\title{
Effect of Electron Beam on Nanoparticle Dynamics in Solution during in situ TEM Observation
}

Jingyu Lu ${ }^{1,2,3,4}$, Zainul Aabdin ${ }^{1,2,3,4}$, Utkarsh Anand ${ }^{3}$, and Utkur Mirsaidov ${ }^{1,2,3,4}$

1. Department of Physics, National University of Singapore, 2 Science Drive 3, Singapore, 117551.

2. Centre for Advanced 2D Materials and Graphene Research Centre, National University of Singapore, 6 Science Drive 2, Singapore 117546.

3. Centre for BioImaging Sciences, Department of Biological Sciences, National University of Singapore, Science Drive 4, Singapore, 117543.

4. Nanocore, 4 Engineering Drive 3, National University of Singapore, Singapore 117576.

The recent development in in situ TEM enabled to visualize the nucleation, growth, coalescence and shape evolution of nanoparticles [1-4]. Some of these processes are driven by electron beam effects [5].

In this study we found that under intense electron beam nanoparticles aggregate together and form a large cluster that relaxes into a large spherical nanoparticle. While under low electron beam different attachment of nanoparticles results in nanorod structures.

The observation was performed with the TEM JEOL 2010F operated at $200 \mathrm{kV}$, and dynamics were captured at 10 frames per second. The sample was made of $1 \mathrm{mM} \mathrm{HAuCl} \mathrm{H}_{4}$ and CTAB solution entrapped between two $20 \mathrm{~nm}$ thick $\mathrm{SiN}_{\mathrm{x}}$ membranes in a liquid cell, more details can be found elsewhere[6]. Attachment of nanoparticles, observed in these liquid cells with TEM for low electron beam condition, is a common way to form nanorod structures as shown in Figure 1. When electron dose is less than $200 \mathrm{e} /\left(\mathrm{A}^{2} . \mathrm{s}\right)$, only small nanoparticles (radius $<3 \mathrm{~nm}$ ) exhibit evident motion (Figure 1(a)(b)). As the dose rate increases, larger nanoparticles start moving (Figure 1(c)-(f)). Note that the nanoparticles encircled in red ellipses in each frame merged together. Two large elongated nanoparticles, formed from individual particles, attach at their endpoints and new longer rod-like nanoparticle is formed. Once these rod-like structures were formed, the attachment of new nanoparticles takes place at the rod tips, thus the nanorod grows even longer, as shown in Figure 1(d)-(f). Similar phenomena are found in the synthesis of $\mathrm{Pt}_{3} \mathrm{Fe}$ nanorods [7] as well.

At high electron doses, the consecutive attachment of 5 nanoparticles forms a big spherical nanoparticle $(\sim 11 \mathrm{~nm}$ in diameter) instead of a nanorods structure as shown in Figure 2. In each attachment case here, the smaller nanoparticle is fused into the larger nanoparticle; our observations reveal that the larger the two attaching nanoparticles, the longer it takes to form a spherical nanoparticle after the coalescence, (Figure 2(a)-(c) vs Figure 2(f)-(g)). Figure 2(i) shows the evolution of projected area of these nanoparticles. The projected area of each new nanoparticle formed by the attachment of two nanoparticles decreases steadily as they tend to become spherical in shape. On the whole, the total projected area was decreased by $\sim 43 \%$ at the end of the process, indicating that the surface energy minimization contributes greatly to this shape relaxation [3]. Further study is on the way to uncover more details. 
References:

[1] H.-G Liao, D Zherebetskyy, H Xin, et al. Science, 345(2014): p. 916-919.

[2] M H Nielsen, S Aloni, J De Yoreo. Science, 345(2014): p. 1158-1162.

[3] Z Aabdin, J Lu, X Zhu, et al. Nano Letters, 14(2014): p. 6639-6643.

[4] M J Williamson, R M. Tromp, P M Vereecken, et al, Nature Materials 2(2003): p. 532 - 536

[5] N de Jonge, and F M. Ross. Nature Nanotechnology, 6(2011): p. 695-704

[6] J Lu, Z Aabdin, L Duane, et al. Nano Letters, 14(2014): p. 2111-2115.

[7] H.-G Liao, L Cui,S Whitelam, H Zheng. Science, 336(2012): p. 1011-1014.

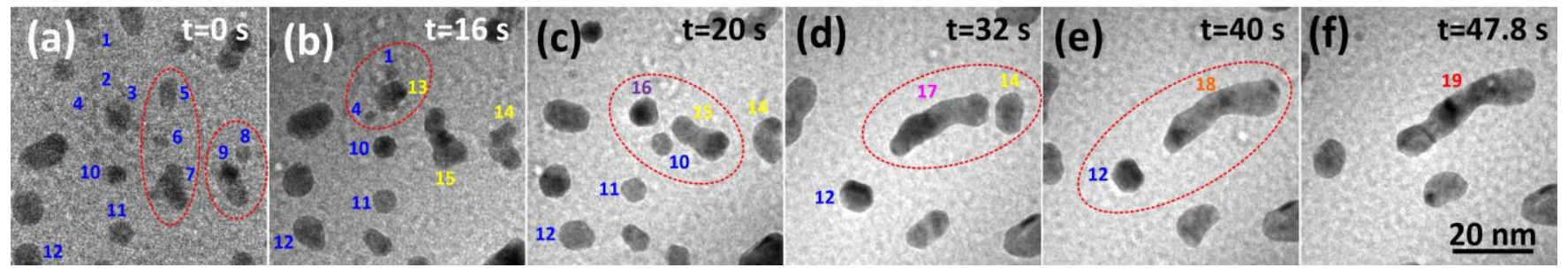

Figure 1. Formation of nanorod by consecutive attachment of nanoparticles. Dose rate in (a) is $~ 60$ $\mathrm{e} / \mathrm{A}^{2} / \mathrm{s}$, and $\sim 1480 \mathrm{e} / \mathrm{A}^{2} / \mathrm{s}$ for (b), and $\sim 4000 \mathrm{e} / \mathrm{A}^{2} / \mathrm{s}$ for (c)-(f). The nanoparticles encircled in red ellipses in each frame merged into a single nanoparticle in the next figure.
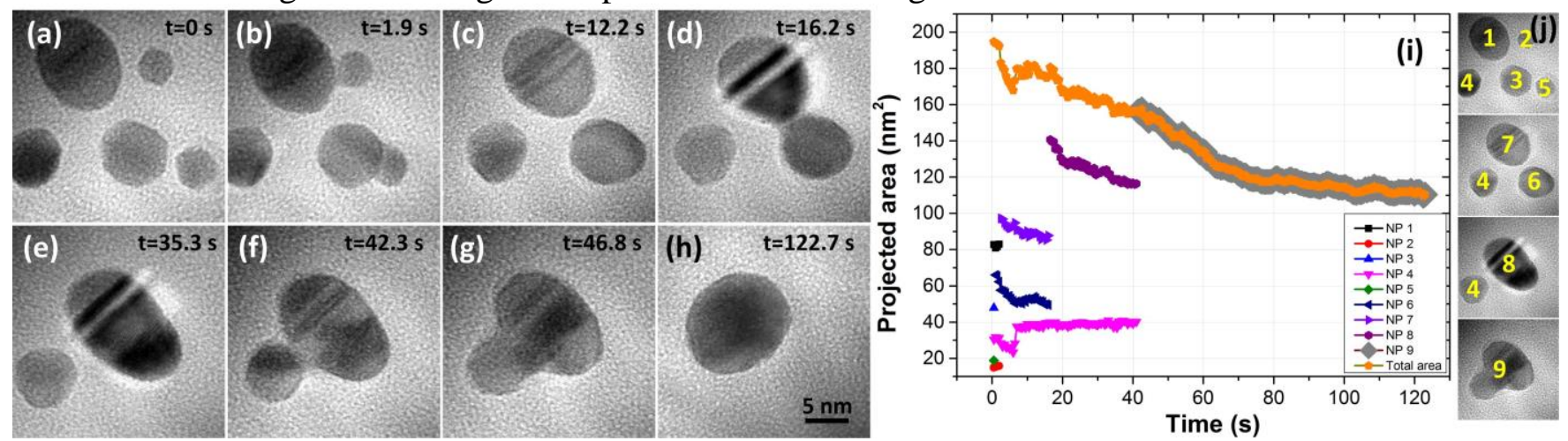

Figure 2. (a)-(h) Formation of a single spherical nanoparticle by consecutive attachment of small nanoparticles. Dose rate: $~ 16000 \mathrm{e} / \mathrm{A}^{2} / \mathrm{s}$. (i) the evolution of projected area of these nanoparticles ("NP" is short for "nanoparticle"), and (j) the corresponding labelled number of nanoparticles. 